\title{
TAGLN expression is upregulated in NF1-associated malignant peripheral nerve sheath tumors by hypomethylation in its promoter and subpromoter regions
}

\author{
GUN-HOO PARK ${ }^{1,2^{*}}$, SU-JIN LEE ${ }^{1,2 *}$, HYUNEE YIM ${ }^{3}$, JAE-HO HAN ${ }^{3}$, HYON J. KIM ${ }^{1}$, \\ YOUNG-BAE SOHN $^{1}$, JUNG MIN KO ${ }^{4}$ and SEON-YONG JEONG ${ }^{1,2}$ \\ Departments of ${ }^{1}$ Medical Genetics, ${ }^{2}$ Biomedical Sciences and ${ }^{3}$ Pathology, Ajou University School of Medicine, Suwon; \\ ${ }^{4}$ Department of Pediatrics, Seoul National University Children's Hospital, Seoul, Republic of Korea
}

Received April 17, 2014; Accepted July 21, 2014

DOI: $10.3892 /$ or.2014.3379

\begin{abstract}
Neurofibromatosis type 1 (NF1) caused by NF1 gene mutation is a commonly inherited autosomal dominant disorder. Malignant peripheral nerve sheath tumors (MPNSTs), a type of aggressive sarcoma, are a major cause of mortality in NF1 patients. The malignant transformation of benign plexiform neurofibromas (PNs) to MPNSTs is a marked peculiarity in NF1 patients, yet the pathogenesis remains poorly understood. We found that an actin-associated protein transgelin (SM22) was highly expressed in NF1-deficient MPNST tissues compared to NFl-deficient PN tissues using immunohistological staining and primary cultured MPNST cells in western blot analysis. We further found that this transgelin upregulation was caused by increased transcriptional expression of the TAGLN gene encoding transgelin. Comparison of DNA methylation values in the promoter and subpromoter regions of the TAGLN gene in three types of NF1-deficient primary-cultured cells, derived from an NF1 patient's normal phenotype, a benign PN and MPNST tissues, revealed that the TAGLN gene was hypomethylated in the MPNST cells. Next, to determine the functional role of transgelin in MPNST pathogenesis, we manipulated the TAGLN gene expression and investigated the alteration of the RAS-mitogen-activated protein kinase (MAPK) signaling pathway in the normalphenotypic and malignant tumor cells. The downregulation
\end{abstract}

Correspondence to: Dr Jung Min Ko, Department of Pediatrics, Seoul National University Children's Hospital, Seoul 110-769, Republic of Korea

E-mail: n-spirit@daum.net

Dr Seon-Yong Jeong, Department of Medical Genetics, Ajou University School of Medicine, San 5, Wonchon dong, Yeongtong-gu, Suwon 443-721, Republic of Korea

E-mail: jeongsy@ajou.ac.kr

${ }^{*}$ Contributed equally

Key words: neurofibromatosis type 1, malignant peripheral nerve sheath tumor, transgelin, TAGLN gene, epigenetics, DNA methylation of TAGLN expression in NF1-deficient MPNST tumor cells through the treatment of the small interfering RNA resulted in a decrease in the RAS activation (GTP-RAS) and the downstream ERK1/2 activation (phosphorylated ERK1/2), while the overexpression of TAGLN in normal-phenotypic NF1-deficient cells caused an increase in RAS and ERK1/2 activation. These results indicate that upregulation of transgelin caused by hypomethylation of the TAGLN gene is closely involved in tumor progression in NF1.

\section{Introduction}

Neurofibromatosis type 1 (NF1; OMIM \#162200) is a commonly inherited autosomal dominant disorder, occurring with an incidence of 1 in 3,000-3,500 individuals worldwide $(1,2)$. NF1 is caused by loss of function mutations in the NF1 gene (GenBank gene ID: NG_009018.1), which encodes a GTPase-activating protein, neurofibromin (3). The major clinical features of NF1 include cafe-au-lait spots, freckling of the axillary or inguinal region, Lisch nodules, optic nerve glioma, bone dysplasias and nerve sheath tumors in the peripheral nervous system, including benign cutaneous neurofibromas, benign plexiform neurofibromas (PNs), and malignant peripheral nerve sheath tumors (MPNSTs) (4).

Since neurofibromin is a major RAS inactivator and plays a role as a tumor suppressor, the lack of neurofibromin resulting from NF1 mutation causes disruptions in the RAS-mitogenactivated protein kinase (MAPK) and PI3K-AKT-mTOR signaling pathways, which is implicated in the tumorigenesis and tumor progression of PN to MPNST (5). MPNST, also called malignant schwannoma or neurofibrosarcoma, develops in $8-13 \%$ of NF1 patients (6), and it represents a major cause of mortality in NF1 patients (7). Thus, the pathogenesis of the malignant transformation of PN to MPNST in NF1 patients has attracted considerable attention $(8,9)$. However, the exact molecular mechanisms of MPNST pathogenesis remain unclear.

Bi-allelic loss of $N F 1$ gene function $\left(N F 1^{-/}\right)$due to the somatic loss of heterozygosity ( $\mathrm{LOH}$ ) of the $N F 1$ gene has been reported to be essential for MPNST development (10). However, LOH at the NF1 locus was also found in PNs (11), 
suggesting that other genetic and/or epigenetic alterations may be involved in tumor progression in NF1. Emerging evidence has suggested that additional driver gene mutations and/or expression alterations contribute to the tumor progression of PNs to MPNSTs (5). Mutations in tumor suppressor genes, including $T P 53, C D K N 2 A$ and $R B$ are commonly identified in MPNSTs (12-14). It was also reported that several cell-cycle and signaling regulation genes, including $C D K N 2 A, C D 44$, CXCR4, EGFR, HGF, MET, PDGFR, PDGFRA, RB1, SOX9 and $T P 53$, are deregulated in MPNSTs $(15,16)$. In addition, many differentially expressed genes between benign neurofibromas and MPNSTs, such as KIT, ERBB2, MET, TGFB1, $H G F, T N X B, T N C, M T O R, T S C 2, P T E N, B C L 2 L 1, C D K 4$, $F O X M 1, B U B 1 B, P B K$ and $N E K 2$, have been identified in comparison studies with immunohistochemical, immunoblotting, microarray-based, quantitative reverse transcription PCR-based or comparative genomic hybridization array-based analyses (17-24).

DNA methylation alterations in cancer-related genes and microRNA (miRNA) dysregulation have been critically implicated in the development and progression of many cancers $(25,26)$. Genes encoding miRNA can be epigenetically regulated by DNA methylation or specific histone modifications (26). Recently, the emerging role of miRNAs in the pathogenesis of NF1 tumorigenesis and MPNST development were reported (27). Genome-wide miRNA profiling analyses found a large number of dysregulated miRNAs in MPNSTs, such as miR-34a against TP53 (28), miR-301a, miR-19a and miR-106b against PTEN (29) and miR-21 against PDCD4 (30). However, only a few DNA methylation studies on NF1 have been reported. In studies of miRNAs, genome-wide DNA methylation analysis may be a new strategy for understanding the etiology of MPNST development in NF1.

In the present study, we reported a $T A G L N$ gene encoding an actin-binding transgelin protein as a novel candidate that plays a critical role in NF1-associated MPNST pathogenesis. TAGLN was upregulated in MPNST tissues and cells derived from NF1 patients. Notably, we found that this upregulation was caused by an alteration of the DNA methylation in the $T A G L N$ genes in MPNSTs. Manipulation of TAGLN expression in the NF1-deficient cells demonstrated the key role of transgelin in MPNST pathogenesis. This finding may provide insight into the underlying mechanisms of somatic tumor progression in NF1 on the epigenetic level.

\section{Materials and methods}

Tumor tissue samples. We used the tumor tissues of eight patients diagnosed with NF1 at the Ajou University Hospital according to NF1 diagnostic criteria (31) (Table I). The PN and MPNST tumor samples were obtained through surgical resection at the Ajou University Hospital. In the case of patients P7 and P8, two tumor specimens obtained from surgeries at two different times were used in the present study. The present study was approved by the Institutional Review Board Committee of the Ajou University School of Medicine.

Primary tissue cultured cells and cell lines. We used three types of previously established primary tissue-cultured NFl-deficient cells: normal phenotype tissues (PC-N), benign PN tissues

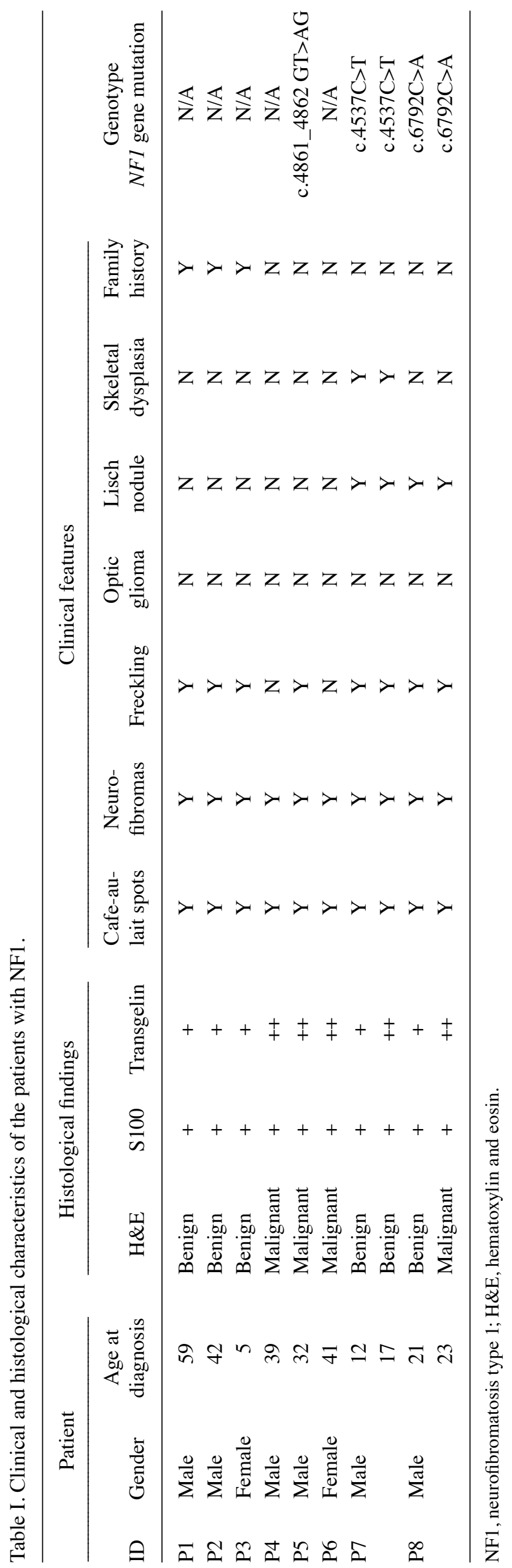


(PC-B) and the MPNST tissues (PC-M) of NF1 patient P8 (32). The cellular characteristics, including GTP-RAS activity and its downstream effectors in the three cell types, were previously demonstrated (32). Cells were grown in Dulbecco's modified Eagle's medium (DMEM) supplemented with $15 \%$ fetal bovine serum (FBS) (both from HyClone Laboratories, Logan, UT, USA), penicillin $(100 \mathrm{U} / \mathrm{ml})$ and streptomycin $(100 \mu \mathrm{g} / \mathrm{ml})$. Cells were used from passages 5 through 10 . The NF1-deficient MPNST cell lines, sNF02.2 and sNF96.2, were purchased from the American Type Culture Collection (Manassas, VA, USA) and grown in DMEM media supplemented with $10 \%$ FBS. All cultured cells were incubated at $37^{\circ} \mathrm{C}$ in a humidified atmosphere containing $5 \% \mathrm{CO}_{2}$.

Hematoxylin and eosin $(H \& E)$ staining and immunohistochemistry (IHC). The tumor tissue specimens from eight patients with NF1 were formalin-fixed and embedded in paraffin wax for pathological evaluation by $\mathrm{H} \& \mathrm{E}$ staining and IHC. Serial $3 \mu \mathrm{m}$ sections were prepared on glass using a cryostat and the slides were stained with H\&E. For IHC, the blocks were cut at $10-\mu \mathrm{m}$ thickness and adhered to glass slides. They were deparaffinized in xylene and rehydrated with graded ethanol, which was followed by antigen retrieval in boiling $10 \mathrm{mM}$ citrate buffer ( $\mathrm{pH}$ 6.0) for $4 \mathrm{~min}$. Immunostaining was carried out using the UltraVision LP Detection System and the HRP Polymer and DAB Plus Chromogen (Thermo Fisher Scientific, Fremont, CA, USA) according to the manufacturer's instructions. Briefly, the sections were incubated with Ultra V Block for $5 \mathrm{~min}$ at room temperature to reduce the nonspecific background, and they were then treated with hydrogen peroxide to block endogenous peroxidase activity. The sections were incubated with the primary antibody for $1 \mathrm{~h}$ at room temperature and then incubated with HRP polymer for 20 min at room temperature. The reaction product was visualized with the DAB chromogen. Pathological evaluation was performed under light microscopy. Anti-transgelin and anti-S100 antibodies were purchased from Thermo Science (Rockford, IL, USA) and Abcam (Cambridge, UK), respectively.

Plasmid constructs and small interfering RNAs (siRNAs). Full-length human TAGLN cDNA was amplified by reverse transcription polymerase chain reaction (RT-PCR) using the primers: 5'-AGTGCAGTCCAAAATCGAGAAG-3' and 5'-CTTGCTCAGAATCACGCCAT-3', which were from the total RNAs of the human skin tissue-cultured fibroblast cells. The cDNAs were subcloned into the pcDNA3.1(-) vector (Clontech, Palo Alto, CA, USA) using the XhoI and BamHI restriction enzyme sites. The siRNAs were synthesized by Genolution Pharmaceuticals, Inc. (Seoul, South Korea). The target sequences for the siRNAs were: 5'-CCAAAATCGA GAAGAAGTATT-3' for the TAGLN gene, and 5'-CCTACGC CACCAATTTCGT-3' for the non-specific scramble siRNA control. Cell transfection of the siRNAs and plasmid constructs was conducted using Lipofectamine RNAiMAX (Invitrogen, Carlsbad, CA, USA) and Lipofectamine 2000 (Invitrogen), respectively, according to the manufacturer's instructions.

Reverse transcription-PCR (RT-PCR). Total RNAs were isolated from the cultured cells using TRIzol reagent and they were treated with RNase-free DNase I (both from Invitrogen) to avoid amplification of the genomic DNA, and were subsequently reverse transcribed using the RevertAid ${ }^{\mathrm{TM}} \mathrm{H}$ Minus First Strand cDNA Synthesis kit (Fermentas, Burlington, ON, Canada) with the oligo(dT) $)_{15-18}$ primer. PCR amplification was carried out using the Ex-Taq DNA polymerase (Takara, Shiga, Japan) at an annealing temperature of $60^{\circ} \mathrm{C}$ for 25 cycles. The gene specific primers used were: 5'-AGTGCAGTCCAA AATCGAGAAG-3' and 5'-CTTGCTCAGAATCACGCCAT-3' for the TAGLN gene; and 5'-TGTTGCCATCAATGACCC CTT-3', and 5'-CTCCACGACGTACTCAGCG-3' for the GAPDH gene (a control).

Western blot analysis. Cultured cells were lysed in RIPA buffer $[150 \mathrm{mM} \mathrm{NaCl}, 1 \%$ Nonidet P-40, 0.5\% sodium deoxycholate, $0.1 \%$ sodium dodecyl sulfate (SDS) and $50 \mathrm{mM}$ Tris buffer, $\mathrm{pH} 8.0]$. The proteins were heated at $100^{\circ} \mathrm{C}$ for $10 \mathrm{~min}$ and analyzed by SDS-polyacrylamide gel electrophoresis on $8-12 \%$ polyacrylamide gels. The proteins were electroblotted onto PVDF membranes (Millipore, Milford, MA, USA). The membrane blots were blocked with $5 \%(w / v)$ non-fat dried milk, incubated with primary and secondary antibodies, and then visualized with the enhanced chemiluminescence western blotting detection system (WEST-ZOL plus; iNtRON Biotechnology, Daejeon, Korea). Anti-transgelin was purchased from Abcam, while the anti-extracellular regulated kinase (ERK)1/2 and anti-phosphorylated ERK1/2 antibodies were purchased from Cell Signaling Technology (Danvers, MA, USA). Anti- $\alpha$-tubulin, HRP-conjugated goat anti-rabbit IgG and HRP-conjugated goat anti-mouse IgG antibodies were purchased from Santa Cruz Biotechnology (Santa Cruz, CA, USA).

Ras activation assay. Ras activation was assayed using the RAS activation assay kit (Upstate Biotechnology, Lake Placid, NY, USA). Briefly, cells were lysed in lysis buffer (25 mM HEPES $\mathrm{pH} 7.5,150 \mathrm{mM} \mathrm{NaCl}, 1 \%$ Igepal CA-630, $10 \mathrm{mM} \mathrm{MgCl}$, $1 \mathrm{mM}$ EDTA, $2 \%$ glycerol, $25 \mathrm{mM} \mathrm{NaF}$ and $1 \mathrm{mM} \mathrm{Na}_{3} \mathrm{VO}_{4}$ ) on ice and centrifuged at $14,000 \mathrm{x}$ g for $5 \mathrm{~min}$ at $4^{\circ} \mathrm{C}$. A total of $300 \mu \mathrm{g}$ cellular lysates were incubated with Raf-RBD agarose beads at $4^{\circ} \mathrm{C}$ for $1 \mathrm{~h}$. The beads were washed 3 times with $1 \mathrm{ml}$ of ice-cold lysis buffer, resuspended in 2X Laemmli sample buffer, boiled and separated on SDS-PAGE gels, which was followed by western blot analysis using an anti-ras antibody.

DNA methylation analysis. DNA methylation analysis was performed using the Infinium HumanMethylation27 BeadChip (Illumina Inc., San Diego, CA, USA; catalog no: WG-311-1201), which contains 27,578 CpG loci located in the promoter and subpromoter regions (5'-UTR, exon 1 and intron 1) of 14,495 human RefSeq genes, as previously described (33). Genomic DNA was isolated from cells using the QIAamp DNA Mini kit (Qiagen, UK). The bisulfite conversion of genomic DNA $(1 \mu \mathrm{g})$ was carried out using the EZ DNA Methylation-Gold kit (Zymo Research, Orange, CA, USA). Bisulfite-converted DNA was amplified and hybridized to the HumanMethylation27 BeadChip according to the manufacturer's standard protocols. Each interrogated locus is represented by specific oligomers linked to two bead types: one representing the sequence for methylated DNA (M) and the other for unmethylated DNA (U). For each specific CpG 

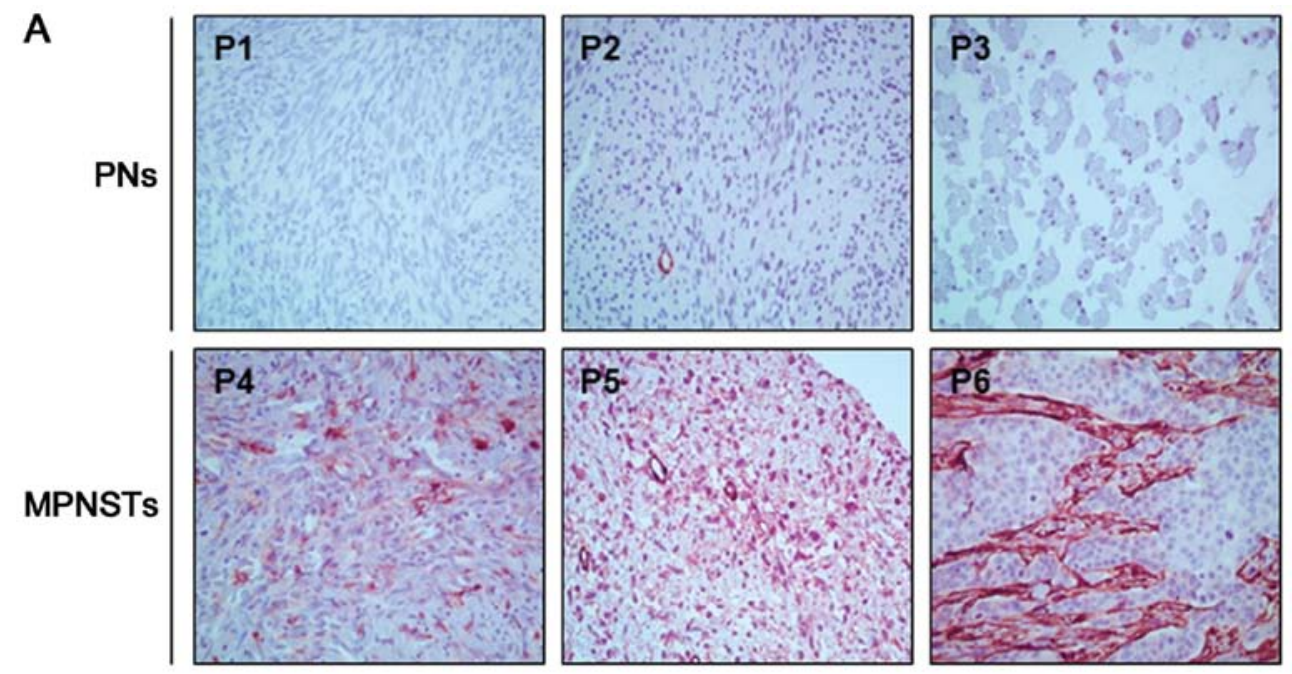

B

Patient 7

Patient 8
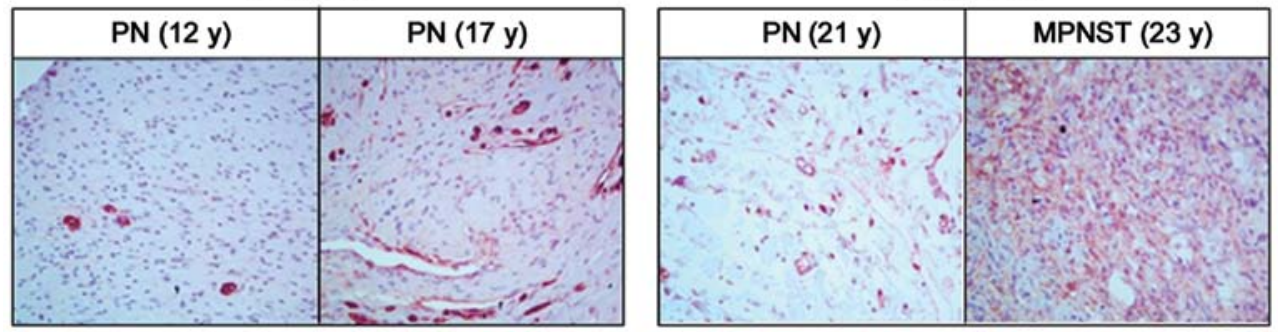

Figure 1. Immunohistochemical staining of transgelin in the plexiform neurofibroma (PN) and malignant peripheral nerve sheath tumor (MPNST) tissues from patients with NF1. (A) Comparison of transgelin levels between tumor tissue sections of PNs (patients P1-P3) and MPNSTs (patients P4-P6). (B) Comparison of transgelin levels in tumor tissue sections from the same patient that were taken at different times. NF1, neurofibromatosis type 1.

island region, the methylation status is calculated from the intensity of the $\mathrm{M}$ and $\mathrm{U}$ alleles, as the ratio of the fluorescent signals $\beta=\operatorname{Max}(M, 0) /[\operatorname{Max}(M, 0)+\operatorname{Max}(\mathrm{U}, 0)+100]$. The DNA methylation $\beta$-value is a way to represent the scores of DNA methylation. Quantitative scores of DNA methylation levels range from 0 (methylation absent) to 1 (completely methylated).

\section{Results}

Upregulation of transgelin in MPNST tissues and primary MPNST cells from NF1 patients. Based on the analysis of the RT-PCR-based differential display data of the normal phenotype, benign PN and MPNST tissues of an NF1 patient, we previously found transgelin (SM22) to be significantly upregulated in MPNST tissues (34). Here, we sought to confirm this finding in more tumor tissues of NF1 patients using IHC analysis. The clinical features and genotypes of the eight NF1 patients analyzed in the present study are summarized in Table I. First, the PN and MPNST tumor specimens of eight NF1 patients were evaluated using histopathological analysis with H\&E staining (Table I). Since Schwann cells are the primary pathogenic cell source in PN and MPNST tumors, we further evaluated tumor specimens through IHC analysis using the Schwann cell lineage marker S100 (Table I).

We compared the transgelin protein levels between the PN and MPNST tumor tissues using IHC analysis. The expression levels of transgelin were higher in the MPNST tissues (P4-P6) than in PN tissues (P1-P3) (Fig. 1A). Next, to investigate whether the transgelin expression level was altered in accor- dance with tumor progression, we compared the transgelin levels in the two tumor tissues resected at different times from the same patient. In patient P7, different transgelin levels were observed in two PN tumors with a five-year interval; the latter showed higher transgelin levels than the former (Fig. 1B). In patient P8, we compared the PN tumor from the first surgery and the MPNST tumor from the second surgery (a 2-year interval) and found significantly higher transgelin expression in the MPNST tumor than in the PN tumor (Fig. 1B).

Subsequently, we confirmed this result in the primary NF1-deficient cells from NF1 patient P8 (32): normalphenotypic cells (PC-N), benign PN cells (PC-B) and MPNST cells (PC-M). Western blot analysis revealed that transgelin expression gradually increased from PC-N to PC-M (Fig. 2A). To determine whether the differential expression of transgelin among the cells was responsible for the changes in the transcriptional expression of the TAGLN gene, we examined the TAGLN mRNA levels by RT-PCR. Consistent with the results for the protein level, the mRNA expression level of TAGLN also gradually increased from PC-N to PC-M (Fig. 2A).

Hypomethylation in the promoter and subpromoter regions of the TAGLN gene in primary MPNST cells from NF1 patients. Since DNA methylation alteration of the TAGLN gene was reported in cancers $(35,36)$, we attempted to investigate the TAGLN gene methylation level in the primary NF1-deficient cells. Using the HumanMethylation27 BeadChip microarray, we performed genome-wide DNA methylation analysis. Following analysis of the methylation data of $27,578 \mathrm{CpG}$ loci 
A

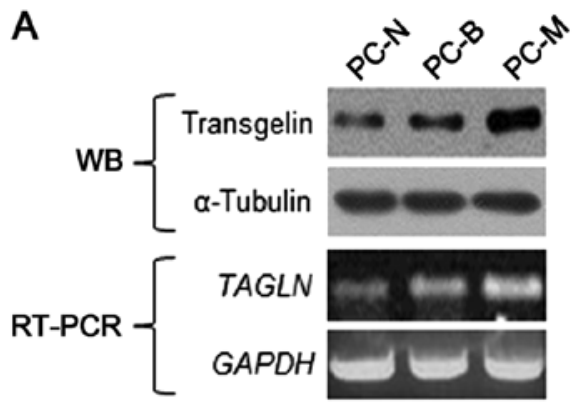

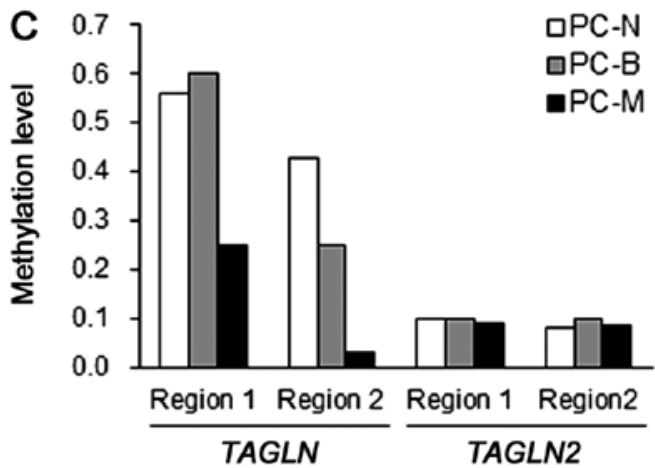

TAGLN TAGLN2

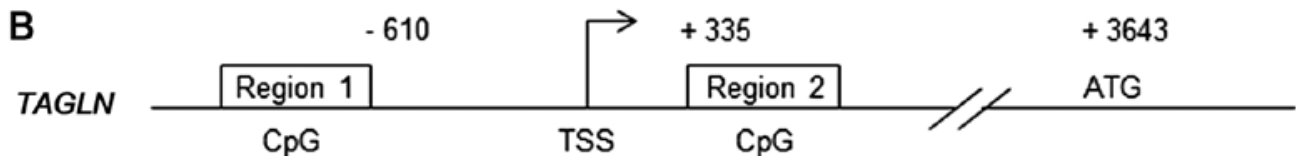

Figure 2. Comparison analyses of the gene expression level and the DNA methylation level of TAGLN in three types of primary NF1-deficient cells. Primarycultured normal-phenotypic cells (PC-N), benign PN cells (PC-B) and MPNST cells (PC-M) from NF1 patient P8 were used in this study. (A) Western blot analysis of transgelin and RT-PCR analysis of TAGLN gene in the three primary cells. (B) Structure of the promoter and subpromoter regions of the TAGLN gene. Two $\mathrm{CpG}$ island regions (region 1 and 2) based on the HumanMethylation27 BeadChip data, transcription start site (TSS), and translation initiating ATG site are indicated with the corresponding cDNA position. (C) DNA methylation status of the TAGLN and TAGLN2 genes in the three primary cells. DNA methylation $\beta$-values of the TAGLN and TAGLN2 genes from the HumanMethylation27 BeadChip microarray are represented as scores from 0 (no methylation) to 1 (complete methylation). NF1, neurofibromatosis type 1; PN, plexiform neurofibroma; MPNST, malignant peripheral nerve sheath tumor.

A

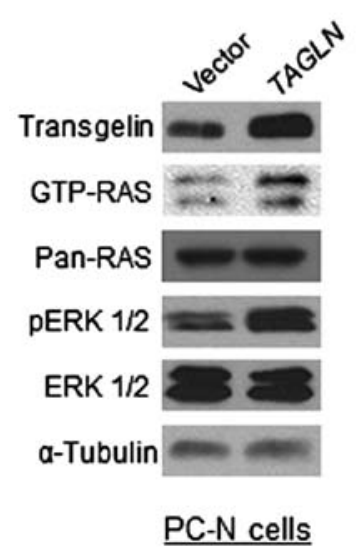

B

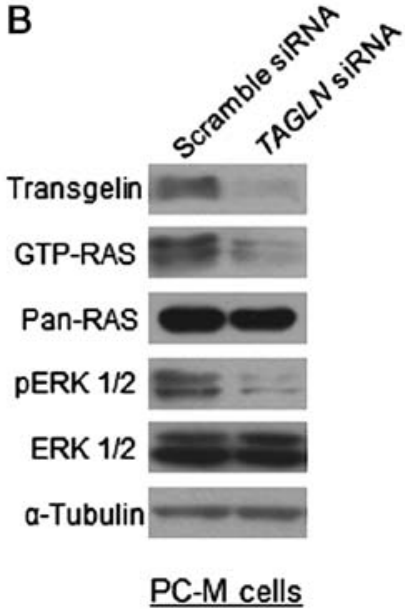

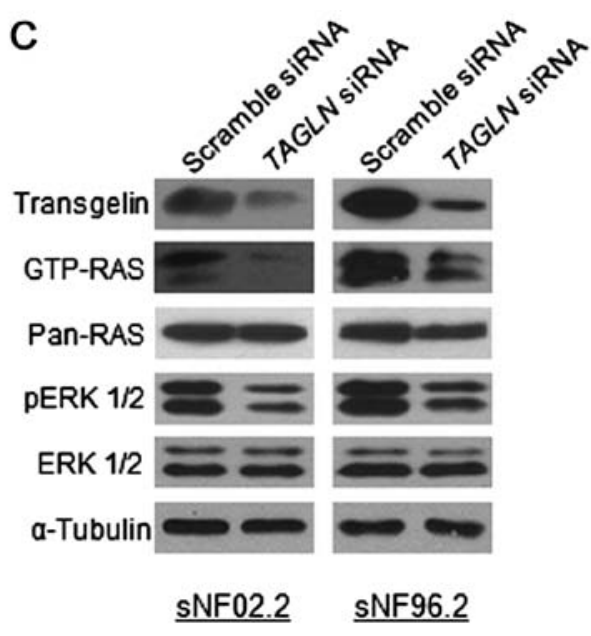

Figure 3. Transgelin level-dependent alterations of RAS and ERK1/2 activation in NF1-deficient primary cells and MPNST cell lines. (A) Overexpression of TAGLN in primary-cultured normal-phenotypic cells (PC-N) from NF1 patient P8. PC-N cells were transfected with a pCDNA3.1 plasmid vector or a TAGLN construct and then incubated for $24 \mathrm{~h}$. (B) Knockdown of TAGLN using small interfering RNAs (siRNAs) in primary-cultured MPNST cells (PC-M) from NF1 patient P8. PC-M cells were treated with TAGLN siRNAs $(50 \mathrm{nM})$ or non-specific scramble control siRNAs (50 nM) and incubated for $72 \mathrm{~h}$. (C) Knockdown of TAGLN using siRNAs in MPNST cell lines, sNF02.2 and sNF96.2. Cells were treated with TAGLN siRNAs (50 nM) or nonspecific scramble control siRNAs $(50 \mathrm{nM})$ and incubated for $72 \mathrm{~h}$. The protein levels of the transgelin, GTP-RAS, total RAS (pan-RAS), phosphorylated ERK1/2 (pERK1/2), ERK1/2 and $\alpha$-tubulin were determined by western blotting. The $\alpha$-tubulin protein level was used as an internal control. NF1, neurofibromatosis type 1 ; MPNST, malignant peripheral nerve sheath tumor.

located in the promoter and subpromoter regions of 14,495 human genes (data not shown), we intensively examined the methylation status of two $\mathrm{CpG}$ island regions in the TAGLN gene (Fig. 2B). We also examined the DNA methylation status of the TAGLN2 gene, which is closely related to TAGLN (37), as a comparison control. The results revealed that both $\mathrm{CpG}$ island regions, regions 1 and 2, in the TAGLN gene were less methylated in the primary PC-M cells than in the PC-N and PC-B cells (Fig. 2C). The DNA methylation level in region 2 of the TAGLN gene gradually decreased from PC-N to PC-M.
In particular, region 2 of the PC-M cells was extremely hypomethylated. In contrast, the DNA methylation status of the TAGLN2 gene was not different in the three types of cells (Fig. 2C). These results indicate that the upregulation of transgelin in the MPNSTs was caused by the hypomethylation of the TAGLN gene.

Involvement of transgelin in RAS and ERK1/2 activation in primary MPNST cells and MPNST cell lines. To examine the functional role of transgelin in MPNST pathogenesis, we 
manipulated the TAGLN gene expression and investigated if the RAS signaling was altered according to the transgelin expression level in the normal-phenotypic cells and malignant tumor cells. Overexpression of TAGLN in the primary PC-N cells caused an increase in Ras activation (GTP-RAS) and the downstream ERK1/2 activation (phosphorylated ERK1/2, pERK1/2) (Fig. 3A). In contrast, the downregulation of TAGLN expression in the primary PC-M cells through the treatment of the short interfering siRNAs (siRNAs) resulted in a decrease in RAS and ERK1/2 activation (Fig. 3B). Next, we carried out a further downregulation experiment in two Schwann-like MPNST cell lines, sNF02.2 $\left(\mathrm{NF}^{+/-}\right)$and sNF96.2 $\left(\mathrm{NF}^{-/}\right)(38)$, that express a high level of transgelin (Fig. 3C). Both cell lines treated with TAGLN siRNAs showed decreased GTP-RAS and pERK1/2, as in the primary PC-M cells (Fig. 3C). These results indicate that the transgelin level is closely involved with the Ras/Raf/Mek/Erk signaling pathway.

\section{Discussion}

Aberrations of DNA methylation in cancer-related genes play a key role in cancer development (39). Hypermethylation of the promoter or first exon of tumor suppressor genes causes transcriptional silencing. In contrast, hypomethylation in proto-oncogenes induces transcriptional activation. Hypermethylation could explain the somatic loss of the tumor suppressor gene function without gene mutations in cancer. However, how hypomethylation contributes to carcinogenesis is less clear (40). In addition, DNA methylation is crucially involved in the dysregulation of miRNAs, which are small non-coding RNAs that act as post-transcriptional regulators of target gene expression, in cancer (41).

Only a few methylation studies have been conducted on NF1. A methylation study on the monozygotic twin pairs with NF1 that presented with several discordant features indicated that differences in the methylation patterns of the normal NFI allele in twins may result in $N F 1$ expression difference, thereby causing a modification of the NF1 phenotype (42). Comparing NF1 patients with a low number of cutaneous neurofibromas to those with a high number of cutaneous neurofibromas, using methylation-specific PCR and pyrosequencing, indicated that the promoter methylation of the mismatch repair $\mathrm{MSH} 2$ gene in the blood cells of patients was significantly different (43). Another methylation study on normal Schwann cells and NF1-associated PN tumor samples reported that a low level of methylation in NF1 gene promoters was found in PN tumors (44). In a methylation analysis of MPNSTs, although no significant methylation changes in the $N F 1$ gene promoter were found in the MPNST tissues (45), frequent PTEN promoter methylation was detected in $N F 1$-associated MPNST

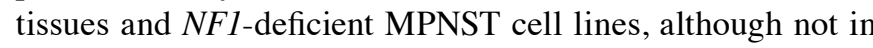
benign tumors (46).

In the present study, we found hypomethylation of the $T A G L N$ gene in the primary MPNST cells and a high expression of transgelin in the MPNST tissues, primary MPNST cells and MPNST cell lines (Figs. 1 and 2). Since the methylation levels in $\mathrm{CpG}$ island region 2 of the TAGLN gene were in inverse proportion to the mRNA expression levels of the TAGLN gene in three types of primary cells (Fig. 2), we concluded that upregulated transgelin in the MPNSTs was a result of the hypomethylation of the TAGLN gene. Although the human promoter region of the TAGLN gene contains two CArG boxes, a binding site for the serum response factor and other transcription factor binding sites for AP-1 and SP1 (47), the epigenetic modification of TAGLN through DNA methylation was also reported to be important in the regulation of transgelin transcription (48). Contrary to our results, however, hypermethylation of TAGLN was found in hepatocellular and colorectal carcinoma $(35,36)$.

Transgelin (SM22) is a 22-kDa actin-binding protein of the calponin family that is found abundantly in the smooth muscle tissues of adult vertebrates (49). Although the precise function of the protein remains unclear, transgelin is suggested to be involved in cell differentiation, cell migration, podosome formation, tissue invasion and matrix remodeling $(50,51)$. Most previous studies reported that transgelin acted as a tumor suppressor (49). However, recent data have indicated that it has a pro-tumorigenic role (50). The upregulation of transgelin was observed in gastric and pancreatic cancers $(52,53)$. Based on these controversial results, the pathological role of transgelin appears to be different between cancer types and could change during tumor progression (50).

Neurofibromas and MPNSTs consist mostly of Schwann cells and fibroblasts and they also contain other cell types, including perineural and mast cells, pericytes, endothelial and smooth muscle cells (54). Fibroblasts are known to express transgelin (53) and Schwann cells also express transgelin based on our data (Fig. 3C), indicating that two of the major cells forming MPNST tumors, Schwann cells and fibroblasts, express transgelin. Thus, the hyperexpression of transgelin in the MPNSTS in the present study may reflect the hyperexpression in these two types of cells, as well as in smooth muscle cells. Schwann cells are well known to contribute fundamentally to MPNST development (55). Fibroblasts are associated with cancer cells in all stages of cancer progression through the production of growth factors, chemokines and the extracellular matrix, and therefore fibroblasts are a key determinant in the malignant progression of cancer (56). It was reported that the upregulation of transgelin in stromal fibroblasts promoted gastric cancer cell migration and invasion by inducing the expression of matrix metalloproteinase-2 (MMP-2) (53). Further studies are required to elucidate how transgelin upregulation in these cells is implicated in tumor progression in NF1.

The molecular mechanisms of transgelin in cancer development remain poorly understood $(49,50)$. The involvement of transgelin in transforming growth factor $\beta$ (TGF- $\beta$ ) and MMP-9 has been reported (49). In addition, RAS-dependent and RAS-independent downregulation of transgelin in human breast and colon carcinomas has been reported (57). Although this study showed results opposite to our results, in that transgelin is downregulated in tumors, it is consistent with our results on how activation of the RAS-RAF-MEK-ERK signaling pathway antagonizes transgelin expression. Our data demonstrated that the upregulation of transgelin in normalphenotypic primary cells by $T A G L N$ overexpression caused an increase in RAS and ERK1/2 activation, while the downregulation of transgelin in MPNST primary cells and cell lines by siRNA treatment resulted in decreased RAS and ERK1/2 activation (Fig. 3). These results indicate that transgelin is closely involved in the RAS-MAPK signaling pathway. 
Our data indicated that transgelin acts as a proto-oncogene rather than as a tumor suppressor gene in NF1-associated MPNST development, which suggests that transgelin may be a suitable target molecule to be inhibited for therapeutic treatment and/or prevention of MPNSTs. Pancreatic cancer patients with high transgelin expression showed a shorter 5-year overall survival rate than those with low transgelin expression (52). Since reduced transgelin expression was accompanied by significantly decreased RAS-MAPK signaling in MPNST cells, the inhibition of transgelin expression may attenuate MPNST development. The upregulation of the miR-145 miRNA enhanced transgelin expression (58); therefore, inhibitors of miR-145 could also be therapeutic drugs in MPNSTs. Gene-specific and genome-wide DNA methylation profiling may be useful biomarkers for diagnosis, risk assessment, prognosis, therapy and therapy-response prediction in cancer $(59,60)$. Recent advances in the field of DNA methylation have begun to elucidate the underlying mechanisms of DNA methylation modification in cancers and to identify novel biomarker targets (60). The methylation profile of the TAGLN gene may be another biomarker that is useful for decision making with regard to the tumor progression of NF1-associated tumors.

\section{Acknowledgements}

This study was supported by a grant (03-2013-0190) from the SNUH Research Fund, and a National Research Foundation of Korea (NRF) grant funded by the Korean government (2014-001483).

\section{References}

1. Boyd KP, Korf BR and Theos A: Neurofibromatosis type 1. J Am Acad Dermatol 61: 1-14, 2009.

2. McClatchey AI: Neurofibromatosis. Annu Rev Pathol 2: 191-216, 2007.

3. Cawthon RM, Weiss R, Xu GF, et al: A major segment of the neurofibromatosis type 1 gene: cDNA sequence, genomic structure, and point mutations. Cell 62: 193-201, 1990.

4. Jett K and Friedman JM: Clinical and genetic aspects of neurofibromatosis 1. Genet Med 12: 1-11, 2010.

5. Katz D, Lazar A and Lev D: Malignant peripheral nerve sheath tumour (MPNST): the clinical implications of cellular signalling pathways. Expert Rev Mol Med 11: e30, 2009.

6. Evans DG, Baser ME, McGaughran J, Sharif S, Howard E and Moran A: Malignant peripheral nerve sheath tumours in neurofibromatosis 1. J Med Genet 39: 311-314, 2002.

7. Grobmyer SR, Reith JD, Shahlaee A, Bush CH and Hochwald SN: Malignant peripheral nerve sheath tumor: molecular pathogenesis and current management considerations. J Surg Oncol 97: 340-349, 2008.

8. Brems H, Beert E, de Ravel T and Legius E: Mechanisms in the pathogenesis of malignant tumours in neurofibromatosis type 1 . Lancet Oncol 10: 508-515, 2009.

9. Thway K and Fisher C: Malignant peripheral nerve sheath tumor: pathology and genetics. Ann Diagn Pathol 18: 109-116, 2014.

10. Upadhyaya M, Kluwe L, Spurlock G, et al: Germline and somatic NF1 gene mutation spectrum in NF1-associated malignant peripheral nerve sheath tumors (MPNSTs). Hum Mutat 29: 74-82, 2008.

11. Kluwe L, Friedrich RE and Mautner VF: Allelic loss of the NF1 gene in NF1-associated plexiform neurofibromas. Cancer Genet Cytogenet 113: 65-69, 1999.

12. Legius E, Dierick H, Wu R, et al: TP53 mutations are frequent in malignant NF1 tumors. Genes Chromosomes Cancer 10 : 250-255, 1994

13. Nielsen GP, Stemmer-Rachamimov AO, Ino Y, Moller MB, Rosenberg AE and Louis DN: Malignant transformation of neurofibromas in neurofibromatosis 1 is associated with CDKN2A/p16 inactivation. Am J Pathol 155: 1879-1884, 1999.
14. Mawrin C, Kirches E, Boltze C, Dietzmann K, Roessner A and Schneider-Stock R: Immunohistochemical and molecular analysis of p53, RB, and PTEN in malignant peripheral nerve sheath tumors. Virchows Arch 440: 610-615, 2002.

15. Upadhyaya M: Genetic basis of tumorigenesis in NF1 malignant peripheral nerve sheath tumors. Front Biosci 16: 937-951, 2011.

16. Mo W, Chen J, Patel A, et al: CXCR4/CXCL12 mediate autocrine cell-cycle progression in NF1-associated malignant peripheral nerve sheath tumors. Cell 152: 1077-1090, 2013.

17. Lévy P, Ripoche H, Laurendeau I, et al: Microarray-based identification of tenascin $C$ and tenascin $X B$, genes possibly involved in tumorigenesis associated with neurofibromatosis type 1 . Clin Cancer Res 13: 398-407, 2007.

18. Watanabe T, Oda Y, Tamiya S, Masuda K and Tsuneyoshi M: Malignant peripheral nerve sheath tumour arising within neurofibroma. An immunohistochemical analysis in the comparison between benign and malignant components. J Clin Pathol 54: 631-636, 2001.

19. Johannessen CM, Reczek EE, James MF, Brems H, Legius E and Cichowski K: The NF1 tumor suppressor critically regulates TSC2 and mTOR. Proc Natl Acad Sci USA 102: 8573-8578, 2005.

20. Park HJ, Lee SJ, Sohn YB, et al: NF1 deficiency causes Bcl-xL upregulation in Schwann cells derived from neurofibromatosis type 1-associated malignant peripheral nerve sheath tumors. Int J Oncol 42: 657-666, 2013.

21. Carroll SL and Ratner N: How does the Schwann cell lineage form tumors in NF1? Glia 56: 1590-1605, 2008.

22. Gregorian C, Nakashima J, Dry SM, et al: PTEN dosage is essential for neurofibroma development and malignant transformation. Proc Natl Acad Sci USA 106: 19479-19484, 2009.

23. Yu J, Deshmukh H, Payton JE, et al: Array-based comparative genomic hybridization identifies $C D K 4$ and FOXM1 alterations as independent predictors of survival in malignant peripheral nerve sheath tumor. Clin Cancer Res 17: 1924-1934, 2011.

24. Stricker TP, Henriksen KJ, Tonsgard JH, Montag AG, Krausz TN and Pytel P: Expression profiling of 519 kinase genes in matched malignant peripheral nerve sheath tumor/plexiform neurofibroma samples is discriminatory and identifies mitotic regulators $B U B 1 B, P B K$ and $N E K 2$ as overexpressed with transformation. Mod Pathol 26: 930-943, 2013.

25. Kulis M and Esteller M: DNA methylation and cancer. Adv Genet 70: 27-56, 2010

26. Malumbres M: miRNAs and cancer: an epigenetics view. Mol Aspects Med 34: 863-874, 2013.

27. Sedani A, Cooper DN and Upadhyaya M: An emerging role for microRNAs in NF1 tumorigenesis. Hum Genomics 6: 23, 2012.

28. Subramanian S, Thayanithy V, West RB, et al: Genome-wide transcriptome analyses reveal p53 inactivation mediated loss of miR-34a expression in malignant peripheral nerve sheath tumours. J Pathol 220: 58-70, 2010.

29. Masliah-Planchon J, Pasmant E, Luscan A, et al: MicroRNAome profiling in benign and malignant neurofibromatosis type 1-associated nerve sheath tumors: evidences of PTEN pathway alterations in early NF1 tumorigenesis. BMC Genomics 14: 473, 2013.

30. Itani S, Kunisada T, Morimoto Y, et al: MicroRNA-21 correlates with tumorigenesis in malignant peripheral nerve sheath tumor (MPNST) via programmed cell death protein 4 (PDCD4). J Cancer Res Clin Oncol 138: 1501-1509, 2012.

31. Ferner RE, Huson SM, Thomas N, et al: Guidelines for the diagnosis and management of individuals with neurofibromatosis 1. J Med Genet 44: 81-88, 2007.

32. Lee SJ, Park HJ, Kim YH, et al: Inhibition of Bcl-xL by ABT-737 enhances chemotherapy sensitivity in neurofibromatosis type 1-associated malignant peripheral nerve sheath tumor cells. Int J Mol Med 30: 443-450, 2012.

33. Bibikova $\mathrm{M}$ and Fan JB: Genome-wide DNA methylation profiling. Wiley Interdiscip Rev Syst Biol Med 2: 210-223, 2010.

34. Jeong SY, Han JH, Park YY and Kim HJ: Identification of differentially expressed genes related to NF1-associated malignant transformation from a patient with neurofibromatosis type 1 . Genes Genomics 30: 407-418, 2008.

35. Hirasawa Y, Arai M, Imazeki F, et al: Methylation status of genes upregulated by demethylating agent 5-aza-2'-deoxycytidine in hepatocellular carcinoma. Oncology 71: 77-85, 2006.

36. Zhao L, Wang H, Deng YJ, et al: Transgelin as a suppressor is associated with poor prognosis in colorectal carcinoma patients. Mod Pathol 22: 786-796, 2009. 
37. Stanier P, Abu-Hayyeh S, Murdoch JN, Eddleston J and Copp AJ: Paralogous sm $22 \alpha$ (Tagln) genes map to mouse chromosomes 1 and 9: further evidence for a paralogous relationship. Genomics 51: 144-147, 1998.

38. Li Y, Rao PK, Wen R, et al: Notch and Schwann cell transformation. Oncogene 23: 1146-1152, 2004.

39. Grønbaek K, Hother C and Jones PA: Epigenetic changes in cancer. APMIS 115: 1039-1059, 2007.

40. Ehrlich M and Lacey M: DNA hypomethylation and hemimethylation in cancer. Adv Exp Med Biol 754: 31-56, 2013

41. Suzuki H, Maruyama R, Yamamoto E and Kai M: DNA methylation and microRNA dysregulation in cancer. Mol Oncol 6: $567-578,2012$.

42. Harder A, Titze S, Herbst L, et al: Monozygotic twins with neurofibromatosis type 1 (NF1) display differences in methylation of NF1 gene promoter elements, $5^{\prime}$ untranslated region, exon and intron 1. Twin Res Hum Genet 13: 582-594, 2010.

43. Titze S, Peters H, Währisch S, et al: Differential $M S H 2$ promoter methylation in blood cells of Neurofibromatosis type 1 (NF1) patients. Eur J Hum Genet 18: 81-87, 2010.

44. Fishbein L, Eady B, Sanek N, Muir D and Wallace MR: Analysis of somatic $N F 1$ promoter methylation in plexiform neurofibromas and Schwann cells. Cancer Genet Cytogenet 157: 181-186, 2005.

45. Harder A, Rosche M, Reuss DE, et al: Methylation analysis of the neurofibromatosis type $1(N F 1)$ promoter in peripheral nerve sheath tumours. Eur J Cancer 40: 2820-2828, 2004.

46. Bradtmöller M, Hartmann C, Zietsch J, et al: Impaired Pten expression in human malignant peripheral nerve sheath tumours. PLoS One 7: e47595, 2012.

47. Camoretti-Mercado B, Forsythe SM, LeBeau MM, et al: Expression and cytogenetic localization of the human SM22 gene (TAGLN). Genomics 49: 452-457, 1998

48. Yamamura $H$, Masuda $H$, Ikeda W, et al: Structure and expression of the human SM22 $\alpha$ gene, assignment of the gene to chromosome 11, and repression of the promoter activity by cytosine DNA methylation. J Biochem 122: 157-167, 1997.

49. Assinder SJ, Stanton JA and Prasad PD: Transgelin: an actinbinding protein and tumour suppressor. Int J Biochem Cell Biol 41: 482-486, 2009.
50. Dvorakova M, Nenutil R and Bouchal P: Transgelins, cytoskeletal proteins implicated in different aspects of cancer development. Expert Rev Proteomics 11: 149-165, 2014.

51. Dos Santos Hidalgo G, Meola J, Rosa E Silva JC, Paro de Paz CC and Ferriani RA: TAGLN expression is deregulated in endometriosis and may be involved in cell invasion, migration, and differentiation. Fertil Steril 96: 700-703, 2011.

52. Zhou L, Zhang R, Zhang L, et al: Upregulation of transgelin is an independent factor predictive of poor prognosis in patients with advanced pancreatic cancer. Cancer Sci 104: 423-430, 2013.

53. Yu B, Chen X, Li J, et al: Stromal fibroblasts in the microenvironment of gastric carcinomas promote tumor metastasis via upregulating TAGLN expression. BMC Cell Biol 14: 17, 2013.

54. Gottfried ON, Viskochil DH, Fults DW and Couldwell WT: Molecular, genetic, and cellular pathogenesis of neurofibromas and surgical implications. Neurosurgery 58: 1-16, 2006.

55. Carroll SL: Molecular mechanisms promoting the pathogenesis of Schwann cell neoplasms. Acta Neuropathol 123: 321-348, 2012.

56. Kalluri R and Zeisberg M: Fibroblasts in cancer. Nat Rev Cancer 6: 392-401, 2006.

57. Shields JM, Rogers-Graham K and Der CJ: Loss of transgelin in breast and colon tumors and in RIE-1 cells by Ras deregulation of gene expression through Raf-independent pathways. J Biol Chem 277: 9790-9799, 2002.

58. Adammek M, Greve B, Kässens N, et al: MicroRNA miR-145 inhibits proliferation, invasiveness, and stem cell phenotype of an in vitro endometriosis model by targeting multiple cytoskeletal elements and pluripotency factors. Fertil Steril 99: 1346-1355.e5, 2013.

59. Vairaktaris E, Kalokerinos G, Goutzanis L, et al: Diabetes enhances cell proliferation but not Bax/Bcl-2-mediated apoptosis during oral oncogenesis. Int J Oral Maxillofac Surg 37: 60-65, 2008.

60. Sinčić N and Herceg Z: DNA methylation and cancer: ghosts and angels above the genes. Curr Opin Oncol 23: 69-76, 2011. 